\title{
Caffeine and Creatine Use in Sport
}

\author{
Mark A. Tarnopolsky \\ Department of Pediatrics and Medicine, McMaster University, Hamilton, Ont., Canada
}

\section{Key Words}

Trimethylxanthine $\cdot$ Coffee $\cdot$ Ergogenic aid $\cdot$ Guanidine compound $\cdot$ Creatine monohydrate

\begin{abstract}
Background/Aims: Caffeine and creatine are 2 of the most widely available and used compounds in sport. Although the use of either is not considered a doping infraction, the evidence does suggest ergogenic potential in certain sports. The purpose of this paper is to review the pharmacology and potential mechanism(s) of action of caffeine and creatine as they pertain to possible use as an ergogenic aid in sport. Methods: Previous review articles on caffeine and creatine use in sport were screened for relevant information and references, and studies for review and recent articles (2007 onwards) were obtained and reviewed using a PUBMED search with the terms 'caffeine AND exercise', 'creatine and creatine monohydrate AND exercise', and appropriate linked articles were evaluated. Results: Caffeine taken before $(3-6 \mathrm{mg} / \mathrm{kg}$ ) or during (1-2 mg/kg) endurance exercise enhances performance, through central nervous system and direct muscle effects. Creatine monohydrate supplementation at higher (approx. 20 g/day $\times 3-5$ days) or lower (approx. 5 g/day $\times$ 30 days) doses increases skeletal muscle total and phosphocreatine by $10-20 \%$. Creatine supplementation appears to minimally but significantly enhance high-intensity sport
\end{abstract}

(C) 2011 S. Karger AG, Basel

$0250-6807 / 10 / 0576-0001 \$ 26.00 / 0$

Fax +4161306 1234

E-Mail karger@karger.ch

www.karger.com
Accessible online at:

www.karger.com/anm performance and the mass and possibly strength gains made during resistance exercise training over the first few months. Conclusions: Although caffeine and creatine appear to be ergogenic aids, they do so in a sport-specific context and there is no rationale for their simultaneous use in sport. Higher doses of caffeine can be toxic and appear to be ergolytic. There is no rationale for creatine doses in excess of the recommendations, and some athletes can get stomach upset, especially at higher creatine doses.

Copyright $\odot 2011$ S. Karger AG, Basel

\section{Introduction}

Caffeine and creatine are 2 widely used compounds in sport and their use is not currently considered to be a doping infraction in spite of the fact that they appear to be ergogenic to certain types of sport performance in certain athletes (note: all athletes should check up-to-date guidelines for their sport as these are subject to change at any time). Part of the difficulty in classifying caffeine or creatine as doping agents is that both of these are found naturally in a wide variety of foods and beverages. As such, it is possible for athletes to get $5 \mathrm{mg} / \mathrm{kg}$ of caffeine from coffee and $5 \mathrm{~g}$ of creatine from certain types of fish or meat, albeit at fairly high intakes. Clearly, although caffeine and creatine are biologically active compounds 
Table 1. Caffeine content of some common foods

\begin{tabular}{lll}
\hline Food/beverage & Serving size & Caffeine content, mg \\
\hline Coffee & $250 \mathrm{ml}$ & \\
$\quad$ Brewed & $250 \mathrm{ml}$ & $100-150$ \\
$\quad$ Drip & $250 \mathrm{ml}$ & $125-175$ \\
$\quad$ Instant & $250 \mathrm{ml}$ & $50-70$ \\
$\quad$ Espresso & 1 shot & $50-110$ \\
Tea & & \\
$\quad$ Green (medium) & $250 \mathrm{ml}$ & $25-40$ \\
$\quad$ Black (medium) & $250 \mathrm{ml}$ & $40-60$ \\
Cola drinks & $355 \mathrm{ml}$ & $35-50$ \\
Energy drinks & $250 \mathrm{ml}$ & $80-150$ \\
Chocolate & & \\
$\quad$ Dark & $50 \mathrm{mg}$ & $20-40$ \\
$\quad$ Milk & $50 \mathrm{mg}$ & $8-16$ \\
\hline
\end{tabular}

The values are a range and some products could be outside the range provided as a function of brewing time and other factors. For example, an analysis of 97 espresso shots taken from retail stores in Australia showed a range of 24-214 mg/shot [61].

that follow all aspects of pharmacology (pharmacokinetics, cellular biological effects, etc.), their presence in foods traditionally renders them as 'nutraceuticals' and not pharmaceuticals. The latter designation is clearly semantic for both caffeine and creatine would be considered pharmacological agents if they were conceived and synthesized by a pharmaceutical company.

The purpose of the current review is to provide an overview of the pharmacokinetics and mechanism(s) of action, and to review the evidence for an ergogenic (workenhancing) effect from both caffeine and creatine in a sport-specific context. It is important for athletes to remember that there are huge interindividual differences that can influence the ergogenic effects of any compound and the potential side effects. Consequently, any athlete must consider the ethical, safety and scientific evidence for an ergogenic benefit of caffeine or creatine in their particular sport. Along these lines, I would recommend an athlete who is considering caffeine or creatine as an ergogenic aid to first try them before an important competition to see if the benefit (ergogenic potential) exceeds the risk (caffeine - tremor, creatine - gastrointestinal upset). There are a number of reviews of both caffeine [1-3] and creatine [4-7] published in the past, and the current review is an update on the topic, including new information since 2008. I do not promote or accept the use of either of these compounds in athletes but it is an individual decision to use either in sport.

\section{Caffeine}

\section{Overview}

Caffeine is an alkaloid xanthine derivative (1,3,7-trimethylxanthine) found in, and added to, a wide variety of foods and beverages (table 1). Most of the caffeinated beverages consumed throughout the world contain caffeine extracted from coffee beans or tea leafs, but caffeine is also eaten as chocolate derived from the cacao bean. Coffee and tea contain a wide variety of other chemicals with potentially bioactive properties including other alkaloids (theobromine, paraxanthine, theophylline) and polyphenols [tannins and flavonoids (chlorogenic acid)]. Consequently, the consumption of caffeine-containing tablets, drinks and other supplements cannot be directly equated to consumption of tea and coffee, from both a health (see below), and possibly ergogenic [1] perspective. Caffeine has been consumed in various foods and beverages for centuries due to its perceived work-enhancing (ergogenic) and alertness effects. Caffeine consumption also has a variety of other dose-dependent effects in the nonhabitual consumer including tachycardia, a slight increase in blood pressure, diuresis, and a fine tremor.

Caffeine is absorbed with a time to peak plasma concentration of 30-90 min and a half-life of about $5 \mathrm{~h}$. Caffeine is metabolized by the cytochrome P450 oxidase system to a variety of xanthine metabolites [8], with about $1-3 \%$ excreted in the urine as free caffeine [3]. The latter fact is important from a drug testing perspective, for it is the free caffeine that is monitored for doping testing. Factors such as hepatic dysfunction, cytochrome P450 single-nucleotide polymorphisms [9], oral contraceptive use, and other drugs can alter metabolism and excretion of metabolites. The habitual consumption of caffeine also influences metabolism by downregulating many of the physiological effects (tachyphylaxis). For example, several days of habitual caffeine consumption attenuated acute ingestion-induced rise in heart rate or blood pressure [10], and the lipolytic effects were attenuated in women during exercise [11]. In contrast to the above data, the ergogenic effects of caffeine are similar in both nonhabitual and habitual caffeine consumers [12].

The International Olympic Committee previously had caffeine on the banned substance list with urinary concentrations greater than $12 \mathrm{mg} / \mathrm{l}$ considered a doping infraction; however, the World Anti-Doping Agency has subsequently removed it in 2004. A potential reason for removal from the banned list could be the fact that low doses of caffeine have been shown to be ergogenic and these levels could be obtained with the habitual con- 
sumption of many common foodstuffs (table 1). In addition, it is the free caffeine that is used for monitoring and this variable shows a manifold interindividual variance in urine concentration for the same ingested dose.

\section{Mechanism(s) of Action}

Caffeine was thought to reveal its ergogenic effects by increasing intracellular cyclic AMP by phosphodiesterase inhibition; however, this concept came from studies using supraphysiologic concentrations (mM) that were not tolerable or likely even possible in humans $(\mu \mathrm{M})$ [3]. The current evidence indicates that caffeine acts through its role as an adenosine receptor antagonist [3]. Adenosine receptors are widely present in human tissue including the brain, skeletal muscle and adipose tissue. Adenosine antagonism in adipocytes clearly leads to increased lipolysis and an increase in plasma free fatty acid (FFA) concentration. Consequently, the 'classical' studies evaluating the ergogenic potential of caffeine considered that the finding of a lower respiratory exchange ratio during endurance exercise indicated that caffeine was increasing plasma FFA concentration, muscle uptake and fat oxidation which led to intramuscular glycogen sparing through the Randle effect $[13,14]$. This hypothesis became well established for over a decade, with a later modification that the sparing occurred primarily in the early rest-toexercise transition; however, more recent studies showed no evidence for glycogen sparing after long-term endurance exercise [2]. The increase in plasma FFA consistently observed at rest is due to an adrenergic (epinephrine, norepinephrine) effect on adipocyte lipolysis; however, this effect is overridden by the adrenergic effect of exercise. Powerful evidence against the Randle effect and adrenergic enhancement as the mechanism of action of caffeine came from the finding that patients with high spinal cord lesions have no increase in epinephrine with caffeine or exercise and still show a caffeine-induced ergogenic effect on electrically stimulated cycle ergometer performance [15].

Caffeine is used in vitro and in situ to potentiate calcium release from the sarcoplasmic reticulum and can lead to a muscle caffeine contracture with supraphysiological concentrations [16]. We [17], and others [18], have shown and enhanced force output in humans during lowfrequency electrical muscle stimulation with caffeine, and we have suggested that this effect was due to enhanced calcium release from the sarcoplasmic reticulum [17]. Specifically, we showed that acute caffeine administration $(5 \mathrm{mg} / \mathrm{kg})$ increased low-frequency $(20 \mathrm{~Hz}$, simulating endurance exercise) electrically stimulated muscle force during a fatiguing protocol [17]. Low-frequency fatigue is felt to occur at the level of calcium release from the sarcoplasmic reticulum [19]. In contrast, caffeine did not enhance power output during electrically stimulated fatigue protocols using high-stimulation frequencies (40 $\mathrm{Hz}$, simulating high-intensity contractions) in our study [17], or at several levels of high-frequency stimulation in another study [18]. Fatigue at high-stimulation frequencies leads to a decrease in electrical activity of the muscle due to conduction block from potassium accumulation and this type of high-frequency fatigue is not altered by caffeine [19]. In contrast, the modest increase in plasma potassium concentration that occurs during endurance exercise was attenuated with caffeine consumption [20], and this could potentially reduce conduction block-induced muscle fatigue [3].

In addition to the ergogenic benefits of caffeine directly on skeletal muscle, there are also central (brain and spinal cord) effects from caffeine administration that may enhance exercise performance during endurance exercise (for a review, see Tarnopolsky [15]). A number of studies have shown that the rating of perceived exertion is lower with caffeine administration versus placebo during endurance exercise. In fact, the best known effect of caffeine is the promotion of wakefulness and this may prevent the central perception of fatigue (central fatigue hypothesis). In addition to a reduction in the central perception of fatigue, a reduction in pain has been demonstrated in several studies following caffeine administration [15].

\section{Caffeine and Exercise Studies}

The initial studies in the late 1970s showed that caffeine administration (approx. $5 \mathrm{mg} / \mathrm{kg}$ ) improved endurance cycle performance in open-ended [14] and closedended [13] cycling tasks. Subsequently, many laboratorybased studies have shown either a greater power output for a given period of time (closed-ended studies) or a greater time to exhaustion (open-ended studies). A large number of studies have also found improvements in endurance sports-specific performance including running, cross-country skiing, and cycling (for a review, see Graham [1] and Tarnopolsky [3]). The vast majority of these studies have used caffeine doses in the range of 3-6 mg/ $\mathrm{kg}$ taken approximately $1 \mathrm{~h}$ prior to exercise. More recently, studies have reported that much lower doses of caffeine $(1.0-2.0 \mathrm{mg} / \mathrm{kg})$, especially when taken later during an endurance exercise task, have enhanced performance [21]. These findings are consistent with athlete anecdotes reporting improvements in performance with the 
Table 2. Creatine content of some common foods

\begin{tabular}{lll}
\hline Food & Serving size & Creatine content, $\mathrm{g}$ \\
\hline Herring & $225 \mathrm{~g} / 8 \mathrm{oz}$ & $2.0-4.0$ \\
Beef (lean) & $225 \mathrm{~g} / 8 \mathrm{oz}$ & $1.5-2.5$ \\
Salmon & $225 \mathrm{~g} / 8 \mathrm{oz}$ & $1.5-2.5$ \\
Pork & $225 \mathrm{~g} / 8 \mathrm{oz}$ & $1.5-2.5$ \\
Milk (1\%) & $250 \mathrm{ml} / 8 \mathrm{oz}$ & 0.05 \\
\hline
\end{tabular}

Creatine is converted to creatinine and the creatine content may decrease from the approximate raw values above as a function of time cooking.

consumption of flat cola type products during endurance cycling. Several gels are now being produced with approximately $50 \mathrm{mg}$ of caffeine per serving.

The evidence for an ergogenic effect of caffeine on high-intensity performance is scant compared to the data with endurance tasks. A number of studies have found no improvement in 30-second all-out sprint cycling performance with caffeine administration and there is no evidence of improvement in single-effort strength or power maneuvers [3]. One study has reported improvements in repetitive high-intensity cycling (2-min sprints followed by a 4 - to 5 -min power output test) [22], and this could be related to a decrease in pain perception.

\section{Potential Side Effects and Other Health Issues}

There have been a large number of epidemiological studies looking at the potential health consequences from habitual caffeine consumption with variable reports over the decades suggesting a potential link to cardiovascular disease or certain types of cancer. However, the vast majority of studies do not find any relationship between habitual coffee consumption (3-8 cups per day) and any negative health consequences [23]. The United States Food and Drug Administration considers caffeine to be generally recognized as safe. It is very important to note that there is a difference between the consumption of coffee, tea, chocolate and cocoa and that of caffeine-supplemented products. The critical issue is that coffee and tea contain over 1,000 biological compounds including the polyphenol chlorogenic acid that has potential beneficial effects such as antioxidant properties and slowing of glucose absorption. In contrast, caffeine per se appears to have a negative effect on insulin sensitivity [24], and would be expected to predispose towards type 2 diabetes. From a dietary consumption perspective, there is very strong evidence that consumption of coffee, even in high daily doses, actually lowers the risk of type 2 diabetes [23].

There are also some individuals who are sensitive to the nervousness and tremor that can be induced with acute caffeine consumption and athletes who get particularly nervous or who develop a tremor may wish to avoid caffeine-containing products prior to exercise. The latter point would be particularly important in accuracy sports such as biathlon where tremor may be an ergolytic effect. Caffeine is also very well known to promote wakefulness and consumption too late in the day may have a major insomnia effect that could exacerbate 'prerace jitters'. Caffeine also has a diuretic effect in nonhabitual consumers; however, this is more apparent at rest and studies have demonstrated that there is no dehydrating effect from caffeine consumption during endurance exercise. For the habitual caffeine consumer, it would also be wise not to acutely stop taking caffeine on the morning of exercise for acute caffeine withdrawal can lead to a significant headache which could be ergolytic to performance.

\section{Creatine Monohydrate Supplementation}

\section{Introduction}

Creatine is a guanidine compound synthesized from the amino acids arginine and glycine in the liver and kidney. Arginine and glycine form guanidinoacetate in the kidney using the enzyme arginine-glycine amidinotransferase, and the guanidinoacetate is converted to creatine with methionine-mediated methylation using guanidinoacetate methyltransferase. Creatine is also present in meat and fish (table 2). Standard cooking has a minimal effect on lowering creatine content through conversion to creatinine [25]. Plasma creatine is transported into tissues via an X-linked creatine transporter. Over $90 \%$ of creatine is taken up into skeletal muscle and creatine is found in high concentration in skeletal muscle (130 $\mathrm{mmol} / \mathrm{kg}$ dry muscle mass). Approximately $65 \%$ of intracellular creatine is phosphorylated (phosphocreatine) and the remainder exists as free creatine. In the rest-toexercise transition, phosphocreatine provides a rapid store for the rephosphorylation of ADP and functions as a temporal energy buffer providing the majority of the energy for the first 6-8 s of muscle contraction before anaerobic glycolysis becomes predominant (fig. 1). The creatine kinase reaction also has a buffering role as depicted in figure 1. The temporal energy buffering is also linked to an activation of mitochondrial respiration forming a 
creatine phosphocreatine shuttle called a spatial energy buffer [26].

There are a number of other cellular effects of creatine, including an increase in the expression of a large number of genes possibly due to cell swelling [27]. Some of these other cellular effects may be responsible for the extensive number of studies demonstrating beneficial effects from creatine supplementation in a variety of animal and human models of neurologic disease [28]. The interest in creatine supplementation for sport came from a seminal paper in 1992 where Roger Harris et al. [29] demonstrated that 5 days of oral administration of creatine monohydrate (20 g/days) increased muscle total and phosphocreatine by approximately $15-20 \%$. Subsequent research demonstrated similar increases in creatine and phosphocreatine after 30 days of low-dose ( $3 \mathrm{~g} /$ day) creatine administration and after a loading dose ( $20 \mathrm{~g} /$ day for 6 days) and $2 \mathrm{~g} /$ day for 1 month [30]. The elevated creatine levels in muscle slowly return to normal after 5-8 weeks after cessation of supplementation [30]. The amount of intramuscular creatine varies between individuals with vegan vegetarians having low levels and individuals consuming high fish or meat diets having elevated levels. The maximal muscle concentration of total creatine is about 160 $\mathrm{mmol} / \mathrm{kg} / \mathrm{dry}$ muscle weight [29]. Given that a muscle biopsy would be required to check for creatine loading and that potentially ergogenic elevations could be found for unexplained reasons in some people (genetic variations in creatine transport capacity) or in those consuming high amounts of fish or meat, creatine supplementation is not on the World Anti-Doping Agency banned list.

\section{Creatine Supplementation and Exercise Performance}

Sprint and high-intensity exercise performance relies heavily on the creatine-phosphocreatine system in the first $10 \mathrm{~s}$ of contraction (for a review, see Terjung et al. [5]). Based on the well-known temporal energy buffering capacity of this system, the logical hypothesis was that if creatine stores could be increased, there could be a prolongation of anaerobic energy delivery in brief sprinting activity. The advantage of the phosphocreatine system is that energy can be provided at a rapid rate and, in contrast to anaerobic glycolysis where lactate is produced, the proton is actually buffered from this reaction (fig. 1). The initial studies were focused on high-intensity cycling for $10-30 \mathrm{~s}$ and several showed a slight increase in power output during this time period [31-33] (for reviews, see Kreider [4] and Terjung et al. [5]). The improvement in highintensity cycling performance was in the order of $1-2 \%$ in many studies, and this is particularly apparent with

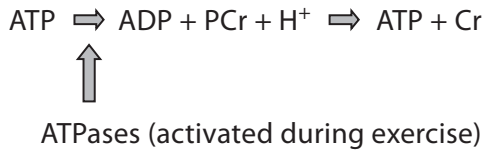

Fig. 1. Creatine-phosphocreatine energy buffering during anaerobic exercise. ATP = Adenosine triphosphate; ADP = adenosine diphosphate; $\mathrm{H}^{+}=$proton (acidity); $\mathrm{PCr}=$ phosphocreatine; $\mathrm{Cr}=$ creatine.

repetitive sprinting activity in a laboratory setting (for reviews, see Kreider [4] and Terjung et al. [5]). Although a couple of percent improvement may seem pedestrian, it should be noted, however, that the difference between the 1st and 8th in many sprinting events at the Olympic level is less than a fraction of a percentage and an enhancement of $1 \%$ could mean the difference between a gold medal and not even making the final. There have been a huge number of studies evaluating cycling, swimming and a host of other high-intensity sporting activities with most, but not all, showing a slight but statistically significant and physiologically relevant improvement in performance [34, 35] (for a review, see Kreider [4]).

Most studies have also reported an acute increase in fat-free mass after 5-7 days of creatine administration (for a review, see Terjung et al. [5]). We have found that this effect is greater in men versus women and the effect in men is approximately $1.5 \mathrm{~kg}$ [36]. This acute increase in fat-free mass is due to an increase in intracellular water; however, in combination with resistance exercise training, the increase in fat-free mass is related to an increase in muscle mass. In fact, the vast majority of studies that have evaluated the co-administration of creatine plus resistance exercise have demonstrated greater improvements in fat-free mass and some measures of strength as compared to resistance training and placebo in both young [37-40] and older [41-43] adults (for a review, also see Terjung et al. [5]).

Conceptually, one would not expect an increase in endurance performance from creatine loading, and the increase in fat-free mass could be ergolytic in weight-supported activities such as running or climbing in cycling. In contrast to the fairly consistent evidence showing that acute creatine supplementation can enhance high-intensity sprinting performance, most studies exhibit no convincing evidence for an ergogenic effect from creatine 
loading (for a review, see Terjung et al. [5]). In fact, one field study showed that creatine administration worsened exercise performance in a cross-country run [44].

\section{Creatine Supplementation and Side Effects/Health}

Issues

There is no supplement in the history of the world that has received more bad press in the lay literature than creatine. Perhaps one of the reasons is that there is a small percentage, but quantitatively large number, of strength/ power athletes who are at risk of taking truly dangerous anabolic drugs and when any negative health consequences occur with them, they look for a more acceptable scapegoat. Another issue I have seen is that the word 'supplement' has a negative connotation and people in general love bad press. For example, I was very surprised when the Lancet and the New England Journal of Medicine readily published anecdotal case reports of 'side effects' from creatine supplementation $[45,46]$. The Lancet paper was on an athlete reportedly taking creatine who had pre-existing renal failure [46], and the New England Journal of Medicine paper reported that a young man got interstitial nephritis after 30 days of creatine supplementation at $20 \mathrm{~g} /$ day) [45]. The subject in the former study was also taking the drug cyclosporine, which is a wellknown nephrotoxic agent, and yet they concluded that creatine, and not the obvious culprit cyclosporine, was the cause. The latter publication indicated that the person was also taking nonsteroidal inflammatory drugs, which are one of the leading causes of interstitial nephritis in young healthy men. Part of the confusion likely comes from the use of serum creatinine as a marker of renal function. Creatine and phosphocreatine are nonenzymatically converted to creatinine within the cell. Since most of the body's creatine is stored inside the muscle, there is a fairly consistent relationship between 24-hour urinary creatinine excretion and muscle mass ( $1 \mathrm{~g}$ creatinine in approx. $20 \mathrm{~kg}$ of muscle). If there is an increase in creatine intake and creatine stores increase, there is a proportionate increase in plasma creatinine due to the increase in delivery to a pool with a normally functioning nephron. Prospective studies using creatine for up to 5 years in healthy people [47-50] and those with disease $[51,52]$ have uniformly shown no adverse effects of creatine supplementation on renal function.

Another very interesting issue was the press that was spread around the world rapidly stating that creatine causes cancer. An investigative report on this anecdote on a French food agency website was touted as a 'clinical trial' by some news agencies and it was subsequently shown that this 'report' was a hyperbole of a highly theoretical speculation based upon nonphysiological organic chemistry involving cooking creatine, creatinine and sugars at high temperatures for long periods [53]. In fact, people are at a far greater risk from this process happening when they charcoal barbeque meats. Unfortunately, the balance of proof for negative press is slim to none and these anecdotes appear to supersede the publications truly evaluating potential side effects (for a review, see Terjung et al. [5]).

Another anecdotal 'side effect' I hear almost daily is that, '...creatine will damage your liver'. I am still not sure where this comes from except perhaps from a report stating that liver transaminases were a bit higher in American football players taking creatine [40]. Again, this misinterpretation is based upon a lack of understanding of physiology for these athletes were also lifting more weights and consequently had higher creatine kinase values, and people often forget that AST and ALT are also found in high abundance in skeletal muscle and cannot be used as a marker of hepatocellular damage in the presence of a high creatine kinase level. We have found that for some reason, creatine monohydrate caused liver inflammation in mice, but not rats [54]. We and others have used $\gamma$-glutamyltransferase and bilirubin as non-muscle markers to clearly show that acute and intermediate (6 months) creatine supplementation had no effect in younger and older humans $[36,43,50]$, or those with neurological disorders [49, 52].

There was some concern that since creatine supplementation led to an increase in intracellular water that this could result in dehydration in athletes. In contrast, several studies have evaluated this concept and found it not to be true and the consensus is that no dehydration occurs [55]. Some, but not all, studies have found that creatine supplementation caused gastrointestinal upset. We have completed many randomized, double-blind studies using creatine and have found that about $5 \%$ of all subjects experience some gastrointestinal upset. However, we have also observed that this side effect is usually related to not dissolving creatine and taking it on an empty stomach, and this is almost invariably eliminated by dissolving or mixing it well (i.e., in yoghurt or other viscous food) and taking it in divided doses at breakfast and supper.

There are an increasing number of papers showing that creatine supplementation may have a beneficial effect in a number of disorders including muscular dystrophy, polymyositis, normal aging, Parkinson's disease, Huntington's disease, brain hypoxia (stroke, cerebral palsy), and congestive heart failure [56, 57]. We and others 
have found that creatine supplementation can increase strength and fat-free mass in children and adults with muscular dystrophy $[51,58]$, and this was confirmed using a meta-analytical approach [59].

\section{Conclusion}

For an athlete wishing to take creatine or caffeine as an ergogenic aid it is important to consider many factors. Firstly, it is important to try anything that you wish to take in competition in training to see how your body reacts to the substance in question. Secondly, although neither creatine nor caffeine are 'banned' substances, it is important to check with current WADA guidelines for ANY substance that a top sport athlete consumes. Thirdly, research studies report the average response and within the population there are responders and nonresponders; consequently, if something does not appear to work for you, do not take it.

Scientifically, the balance of the evidence indicates that the consumption of caffeine at $2-6 \mathrm{mg} / \mathrm{kg}$ before exercise or $0.75-2.0 \mathrm{mg} / \mathrm{kg}$ during exercise will enhance endurance exercise performance. There is no strong evidence that caffeine consumption will enhance high-intensity sprint or strength performance, but certainly no evidence that it would harm performance. Some athletes, especially those who are nonhabitual caffeine consumers, may experience some gastrointestinal upset, pre-exercise diuresis or tremor that may inhibit performance. For habitual caffeine consumers, it would likely not be wise to withhold caffeine in the $8-12 \mathrm{~h}$ before exercise for the withdrawal side effects (headache) may inhibit performance.

The balance of the evidence suggests that creatine loading (5 g 4 times a day for $4-5$ days OR $3 \mathrm{~g} /$ day for 30 days) will enhance repetitive high-intensity sprint performance and may enhance performance in sports with high-intensity bursts of power. Furthermore, most studies do show that creatine administration enhances the strength and muscle mass gains during a resistance exercise program as compared to placebo (at least over the first 4-6 months of a training program). There is no convincing evidence that there is an ergogenic benefit from creatine loading for endurance athletes. Some athletes (approx. 5\%) experience gastrointestinal upset with creatine administration.

Although there is little rationale for taking both caffeine and creatine simultaneously as ergogenic aids, some have reported that the acute consumption of both negated the ergogenic benefits [60].

\section{Disclosure Statemement}

Dr. Tarnopolsky is the co-founder and chief scientific officer of Life Sciences Nutritionals (we do not make any products containing either caffeine or creatine).

\section{References}

1 Graham TE: Caffeine, coffee and ephedrine: impact on exercise performance and metabolism. Can J Appl Physiol 2001;26 (suppl):S103-S119.

- Graham TE, et al: Does caffeine alter muscle carbohydrate and fat metabolism during exercise? Appl Physiol Nutr Metab 2008;33: 1311-1318.

3 Tarnopolsky MA: Caffeine and endurance performance. Sports Med 1994;18:109-125.

4 Kreider RB: Effects of creatine supplementation on performance and training adaptations. Mol Cell Biochem 2003;244:89-94.

$\checkmark 5$ Terjung RL, et al: American College of Sports Medicine roundtable. The physiological and health effects of oral creatine supplementation. Med Sci Sports Exerc 2000;32: 706-717.

6 Tarnopolsky MA: Clinical use of creatine in neuromuscular and neurometabolic disorders. Subcell Biochem 2007;46:183-204.
7 Greenhaff PL: Creatine and its application as an ergogenic aid. Int J Sport Nutr 1995; 5(suppl):S100-S110.

8 Kot M, Daniel WA: Caffeine as a marker substrate for testing cytochrome $\mathrm{P} 450$ activity in human and rat. Pharmacol Rep 2008;60: 789-797.

9 Chen X, et al: The G-113A polymorphism in CYP1A2 affects the caffeine metabolic ratio in a Chinese population. Clin Pharmacol Ther 2005;78:249-259.

10 Robertson D, et al: Tolerance to the humoral and hemodynamic effects of caffeine in man. J Clin Invest 1981;67:1111-1117.

11 Fisher SM, et al: Influence of caffeine on exercise performance in habitual caffeine users. Int J Sports Med 1986;7:276-280.

12 Van Soeren MH, et al: Caffeine metabolism and epinephrine responses during exercise in users and nonusers. J Appl Physiol 1993; 75:805-812.
13 Ivy JL, et al: Influence of caffeine and carbohydrate feedings on endurance performance. Med Sci Sports 1979;11:6-11.

14 Costill DL, Dalsky GP, Fink WJ: Effects of caffeine ingestion on metabolism and exercise performance. Med Sci Sports 1978;10: 155-158.

15 Tarnopolsky MA: Effect of caffeine on the neuromuscular system - Potential as an ergogenic aid. Appl Physiol Nutr Metab 2008; 33:1284-1289.

16 Weber A, Herz R: The relationship between caffeine contracture of intact muscle and the effect of caffeine on reticulum. J Gen Physiol 1968;52:750-759.

17 Tarnopolsky M, Cupido C: Caffeine potentiates low frequency skeletal muscle force in habitual and nonhabitual caffeine consumers. J Appl Physiol 2000;89:1719-1724.

18 Lopes JM, et al: Effect of caffeine on skeletal muscle function before and after fatigue. J Appl Physiol 1983;54:1303-1305. 
19 Westerblad H, Allen DG: Mechanisms underlying changes of tetanic $\left[\mathrm{Ca}^{2+}\right]_{\mathrm{i}}$ and force in skeletal muscle. Acta Physiol Scand 1996; 156:407-416.

20 Lindinger MI, Graham TE, Spriet LL: Caffeine attenuates the exercise-induced increase in plasma $\left[\mathrm{K}^{+}\right]$in humans. J Appl Physiol 1993;74:1149-1155.

21 Cox GR, et al: Effect of different protocols of caffeine intake on metabolism and endurance performance. J Appl Physiol 2002;93: 990-999.

22 Jackman M, et al: Metabolic catecholamine, and endurance responses to caffeine during intense exercise. J Appl Physiol 1996;81: 1658-1663.

23 van Dam RM: Coffee consumption and risk of type 2 diabetes, cardiovascular diseases, and cancer. Appl Physiol Nutr Metab 2008; 33:1269-1283.

24 Robinson LE, et al: Caffeine ingestion before an oral glucose tolerance test impairs blood glucose management in men with type 2 diabetes. J Nutr 2004;134:2528-2533.

25 Harris RC, et al: The concentration of creatine in meat, offal and commercial dog food. Res Vet Sci 1997;62:58-62.

26 Wallimann T, et al: Intracellular compartmentation, structure and function of creatine kinase isoenzymes in tissues with high and fluctuating energy demands: the 'phosphocreatine circuit' for cellular energy homeostasis. Biochem J 1992;281:21-40.

27 Safdar A, et al: Global and targeted gene expression and protein content in skeletal muscle of young men following short-term creatine monohydrate supplementation. Physiol Genomics 2008;32:219-228.

-28 Tarnopolsky MA, Beal MF: Potential for creatine and other therapies targeting cellular energy dysfunction in neurological disorders. Ann Neurol 2001;49:561-574.

29 Harris RC, Soderlund K, Hultman E: Elevation of creatine in resting and exercised muscle of normal subjects by creatine supplementation. Clin Sci (Lond) 1992;83:367-374.

30 Hultman E, et al: Muscle creatine loading in men. J Appl Physiol 1996;81:232-237.

-31 Greenhaff PL, et al: Influence of oral creatine supplementation of muscle torque during repeated bouts of maximal voluntary exercise in man. Clin Sci (Lond) 1993;84:565-571.

32 Birch R, Noble D, Greenhaff PL: The influence of dietary creatine supplementation on performance during repeated bouts of maximal isokinetic cycling in man. Eur J Appl Physiol Occup Physiol 1994;69:268-276.
Casey A, et al: Creatine ingestion favorably affects performance and muscle metabolism during maximal exercise in humans. Am J Physiol 1996;271:E31-E37.

34 Anomasiri W, Sanguanrungsirikul S, Saichandee P: Low dose creatine supplementation enhances sprint phase of 400 meters swimming performance. J Med Assoc Thai 2004;87(suppl 2):S228-S232.

35 Vandebuerie F, et al: Effect of creatine loading on endurance capacity and sprint power in cyclists. Int J Sports Med 1998;19:490495.

36 Mihic S, et al: Acute creatine loading increases fat-free mass, but does not affect blood pressure, plasma creatinine, or CK activity in men and women. Med Sci Sports Exerc 2000;32:291-296.

37 Becque MD, Lochmann JD, Melrose DR: Effects of oral creatine supplementation on muscular strength and body composition. Med Sci Sports Exerc 2000;32:654-658.

- 38 Vandenberghe $\mathrm{K}$, et al: Long-term creatine intake is beneficial to muscle performance during resistance training. J Appl Physiol 1997;83:2055-2063.

39 Jowko E, et al: Creatine and beta-hydroxybeta-methylbutyrate (HMB) additively in crease lean body mass and muscle strength during a weight-training program. Nutrition 2001;17:558-566.

40 Kreider RB, et al: Effects of creatine supplementation on body composition, strength, and sprint performance. Med Sci Sports Exerc 1998;30:73-82.

41 Brose A, Parise G, Tarnopolsky MA: Creatine supplementation enhances isometric strength and body composition improvements following strength exercise training in older adults. J Gerontol A Biol Sci Med Sci 2003;58:11-19.

42 Chrusch MJ, et al: Creatine supplementation combined with resistance training in older men. Med Sci Sports Exerc 2001;33:21112117.

43 Tarnopolsky MA, Safdar A: The potential benefits of creatine and conjugated linoleic acid as adjuncts to resistance training in older adults. Appl Physiol Nutr Metab 2008;33: 213-227.

44 Balsom PD, et al: Creatine supplementation per se does not enhance endurance exercise performance. Acta Physiol Scand 1993;149: 521-523.

-45 Koshy KM, Griswold E, Schneeberger EE: Interstitial nephritis in a patient taking creatine. N Engl J Med 1999;340:814-815.

-46 Pritchard NR, Kalra PA: Renal dysfunction accompanying oral creatine supplements. Lancet 1998;351:1252-1253.
47 Poortmans JR, et al: Effect of short-term creatine supplementation on renal responses in men. Eur J Appl Physiol Occup Physiol 1997; 76:566-567.

48 Poortmans JR, Francaux M: Long-term oral creatine supplementation does not impair renal function in healthy athletes. Med Sci Sports Exerc 1999;31:1108-1110.

49 Tarnopolsky M, et al: Creatine monohydrate and conjugated linoleic acid improve strength and body composition following resistance exercise in older adults. PLoS One 2007;2:e991.

50 Kreider RB, et al: Long-term creatine supplementation does not significantly affect clinical markers of health in athletes. Mol Cell Biochem 2003;244:95-104.

51 Tarnopolsky MA, et al: Creatine monohydrate enhances strength and body composition in Duchenne muscular dystrophy. Neurology 2004;62:1771-1777.

52 Groeneveld GJ, et al: A randomized sequential trial of creatine in amyotrophic lateral sclerosis. Ann Neurol 2003;53:437-445.

53 Wyss M, Kaddurah-Daouk R: Creatine and creatinine metabolism. Physiol Rev 2000;80: 1107-1213.

54 Tarnopolsky MA, et al: Histological assessment of intermediate- and long-term creatine monohydrate supplementation in mice and rats. Am J Physiol Regul Integr Comp Physiol 2003;285:R762-R769.

55 Volek JS, et al: Physiological responses to short-term exercise in the heat after creatine loading. Med Sci Sports Exerc 2001;33:11011108

56 Yang L, et al: Combination therapy with coenzyme Q10 and creatine produces additive neuroprotective effects in models of Parkinson's and Huntington's diseases. J Neurochem 2009;109:1427-1439.

57 Zhu S, et al: Prophylactic creatine administration mediates neuroprotection in cerebral ischemia in mice. J Neurosci 2004;24:59095912

58 Louis M, et al: Beneficial effects of creatine supplementation in dystrophic patients. Muscle Nerve 2003;27:604-610.

59 Kley R, Vorgerd M, Tarnopolsky M: Creatine for treating muscle disorders. Cochrane Database Syst Rev 2007:CD004760.

60 Vandenberghe K, et al: Caffeine counteracts the ergogenic action of muscle creatine loading. J Appl Physiol 1996;80:452-457.

61 Desbrow B, et al: An examination of consumer exposure to caffeine from retail coffee outlets. Food Chem Toxicol 2007;45:15881592. 